\title{
Transforaminal Lumbar Interbody Fusion for Management of Recurrent Lumbar Disc Herniation
}

\author{
Haitham El-Beltagy Abd El-Kader \\ Department of Neurosurgery, Al-Menoufia University, Al-Menoufia, Egypt
}

\section{Study Design: Retrospective study.}

Purpose: To study the surgical outcome of transforaminal lumbar interbody fusion (TLIF) combined with trans-pedicular screws fixation for management of selected cases of recurrent lumbar disc herniation.

Overview of Literature: Recurrent lumbar disc herniation is a major cause of surgical failure, occurring in $5 \%-11 \%$ of cases. The optimal technique for treatment is controversial. Some authors believe that repeated simple discectomy is the treatment of choice, but approach-related complications can be considerable. Other surgeons prefer more removal of posterior elements (as lamina and facet joints) with posterior fusion.

Methods: The study included 15 patients who presented with symptomatic recurrent lumbar disc herniation who underwent reoperation through posterior trans-pedicular screws and TLIF in our department from April 2008 to May 2010, with a 24-month followup. Japanese Orthopedic Association Scale (JOA) was used for low back pain. The results of surgery were also evaluated with the MacNab classification.

Results: The mean JOA score showed significant improvement, increasing from 9.5 before surgery to 24.0 at the end of follow-up $(p<0.001)$. Clinical outcome was excellent in 7 patients ( $46 \%$ of cases), good in 6 patients $(40 \%)$ and fair in only 2 patients $(14 \%)$. There was a significant difference $(p<0.05)$ between patients presenting with recurrent disc at the ipsilateral side and those at the contralateral side.

Conclusions: In spite of the small number of patients and the short follow-up period, the good clinical and radiological outcome achieved in this study encourage the belief that TLIF is an effective option for the treatment of selected cases of recurrent lumbar disc herniation.

Keywords: Recurrent lumbar disc herniation; Transpedicular screws; Transforaminal lumbar interbody fusion

\section{Introduction}

Recurrent lumbar disc herniation is a major cause of surgical failure, with an incidence rate of was $5 \%-11 \%$ in patients undergoing spinal surgical procedures, with an increased incidence as the follow-up period is extended.
How to optimally treat recurrent disc herniation is controversial. Some believe that repeated simple discectomy is the treatment of choice, with clinical outcome similar to the primary procedure. However, approach-related complications can be considerable as scar tissue makes it more difficult, increasing the risk of dural tear or nerve

Received Apr 25, 2015; Revised May 24, 2015; Accepted May 25, 2015

Corresponding author: Haitham El-Beltagy Abd El-Kader

Department of Neurosurgery, Al-Menoufia University, 11 El Sadat st., Shebin El-kom, Al-Menoufia, Egypt

Tel: +20-1003434915 Fax:+20-482221013, E-mail: hithamelbeltagy@yahoo.com 
injury [1]. Some spine surgeons believe that posterior fusion is necessary for treating recurrent disc herniation as repeated discectomy for disc recurrence requires removal of more posterior elements, such as lamina and/or facet joint, to approach the disc space laterally avoiding previous scar tissue around discectomy site, which increases the risk of segmental instability [2]. A large retrospective follow-up study of patients undergoing multiple revisions after lumbar discectomy revealed markedly reduced risk for subsequent operations if the first procedure was a spinal fusion (5.0\% vs. $24.9 \%$ after discectomy and $27.2 \%$ after spinal decompression). Therefore, the use of fusion to treat or prevent segmental instability after repeated discectomy appears to be a reasonable choice in cases of recurrent disc herniation [3].

There are several types of lumbar fusion technique. Those most often used are posterior lumbar fusion (PLF), posterior lumbar interbody fusion (PLIF), anterior lumbar interbody fusion (ALIF), and more recently, the transforaminal lumbar interbody fusion (TLIF) [4].

TLIF is an increasingly popular technique that is used to achieve a circumferential arthrodesis in the lumbar spine. The procedure is similar to PLIF in that it allows fusion without necessitating a separate anterior approach [5]. Compared to PLIF, TLIF offers several advantages. It involves unilateral removal of a facet joint. This more lateral approach to the disc space requires minimal retraction of the dura mater, thus reducing the risk of neurological injury. For the same reason, TLIF also can be performed at the upper lumbar levels where proximity to the conus medullaris makes the dural retraction necessary in PLIF unfeasible [6]. In addition, TLIF can be performed contralateral to the site of a prior laminotomy, thus avoiding adherent scar tissue. The results reported on initial studies of TLIF have been promising; clinical outcomes and fusion rates were comparable to those seen after PLIF or circumferential fusion performed using combined anterioposterior (AP) approaches [7]. The main indications of this surgery are low grade spondylolisthesis, spinal stenosis, recurrent disc herniation and some cases of degenerative disc disease, when solid interbody and posterolateral fusion with posterior decompression is needed [8]. Biomechanically, TLIF provides anterior column support and a posterior tension band. It can be safely performed via a unilateral posterior approach at any vertebral level. Revision can be done through the undisturbed contralateral foramen. TLIF provides adequate surface area for solid anterior fusion [9].

\section{Materials and Methods}

\section{Patients}

This retrospective study included 15 patients ( 9 males, 6 females; mean age at diagnosis, 47.5 years; range, 35-60 years). The patients presented with symptomatic recurrent lumbar disc herniation at different levels as shown in Table 1 and had been treated in the Department of Neurosurgery, Al-Menoufia University from April 2008 to May 2010. Inclusion criteria included persistent postoperative low back pain and unilateral sciatica unresponsive to conservative treatment for at least 3 months, recurrent disc herniation at the same level as previous surgery at the ipsilateral or the contralateral side, at least 6 months of pain relief after primary disc surgery, and medical fitness. Confirmation of diagnosis was done through X-ray radiographs, computed tomography (CT), and magnetic resonance imaging (MRI) with and without contrast (Figs. $1,2)$.

\section{Surgical technique}

After selection of patients and surgical consent, all patients

Table 1. Level of recurrent disc herniation

\begin{tabular}{lc} 
Disc level & No. $(\%)$ \\
L4-5 & $11(74)$ \\
L5-S1 & $3(20)$ \\
L3-4 & $1(6)$ \\
\hline
\end{tabular}

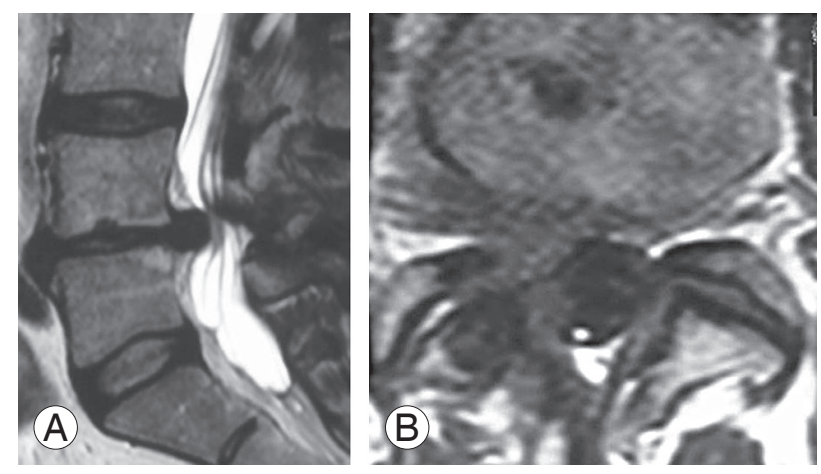

Fig. 1. (A) Preoperative sagittal magnetic resonance imaging (MRI) showing recurrent $L 4-5$ disc herniation. (B) Preoperative axial MRI showing $L 4-5$ disc reherniation and right nerve root compression. 

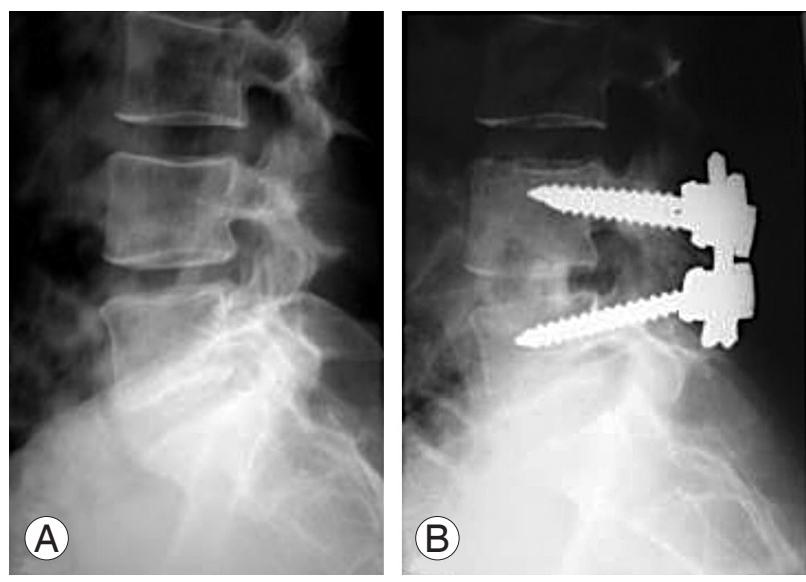

Fig. 2. (A) Preoperative lateral $X$-ray showing narrow $L 4-5$ disc space. (B) Postoperative (3 months) lateral X-ray showing L4-5 transpedicular screws with the transforaminal lumbar interbody fusion technique.

Table 2. Primary procedure done among the studied group

\begin{tabular}{lc} 
Primary surgery & No. $(\%)$ \\
Unilateral hemilaminectomy & $8(53)$ \\
Bilateral (total) laminectomy & $7(47)$ \\
\hline
\end{tabular}

included in the study underwent surgery (Table 2) using the midline posterior approach. Bilateral dissection was extended just lateral to the facet joints and the epidural scar tissue left intact. Trans-pedicular screws were inserted in the usual fashion. On the symptomatic side, the pars interarticularis was removed and a hemifacetectomy performed on the superior and inferior facets at the level of the spinal segment to be fused. Nearly complete discectomy was performed using disc rongeurs and curettes and rongeurs. End-plate decortication was performed. Intervertebral disc space spreaders were then sequentially inserted and rotated to restore the normal disc space height. Once the disc space was distracted, the anterior two-third of the disc space was packed with cancellous bone from the laminectomy bone. A single cage packed with bone was inserted posterolaterally and oriented anteromedially. Finally, connecting rods were placed and compression was applied across the instrumentation to restore segmental lordosis and was locked in place.

\section{Follow-up}

At 3, 6, 12, and 24 months postoperatively, clinical symptoms were assessed at each interval. The Japanese Orthopedic Association (JOA) evaluation system [10] for low-

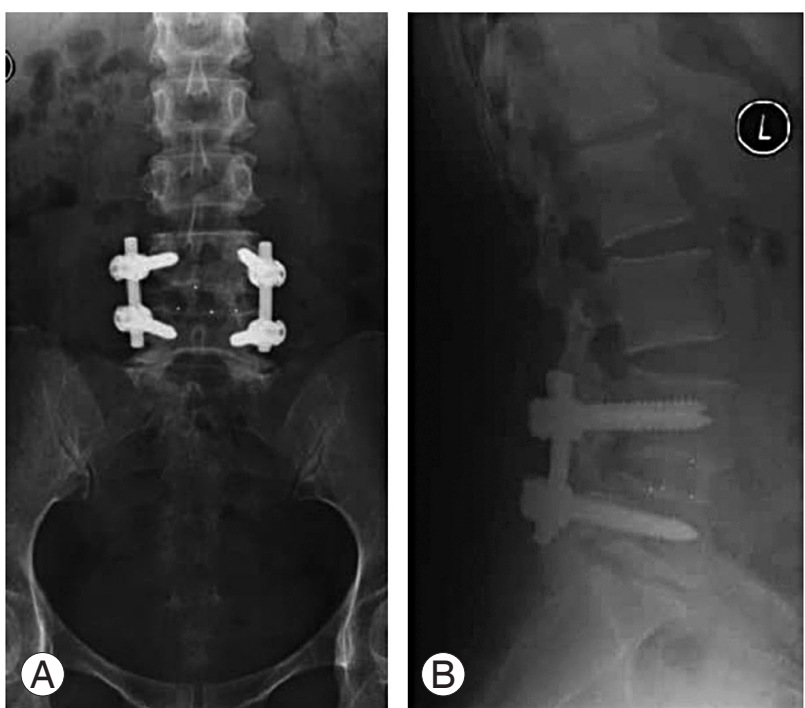

Fig. 3. (A) Postoperative anterioposterior X-ray showing L4-5 solid fusion. (B) Twenty-four month postoperative lateral X-ray showing maintained disc height with stable spine on extension.

back pain syndrome was determined by direct questioning of each patients to assess subjective symptoms, clinical signs, and the restriction of activities of daily living. For radiological assessment AP and lateral X-rays were obtained at the same times, with flexion and extension $\mathrm{X}$-rays done at 12, 24 months (Fig. 3). An independent radiologist evaluated the fusion status based on the radiographs. Criteria for successful fusion were lack of motion, anterior bridging and the lack of lucencies on flexion/extension X-rays, and/or contiguous bone through the cage in a thin-cut sagittal CT scan. Surgical outcome was classified using the MacNab classification [11] with a score of 1 indicating an excellent outcome (improvement $>90 \%$ ), 2 indicating a good outcome (75\%-90\% improvement), 3 indicating fair outcome (50\%-74\% improvement), and 4 indicating poor outcome ( $<49 \%$ improvement).

\section{Results}

In the 15 patients, peak incidence was between 35-45 years of age in 9 patients ( $60 \%$ of total cases) and the lowest incidence was between 25-35 years of age in 2 patients (13\% of total cases). The mean age was 47.5 years. Nine $(60 \%)$ of the patients were males and $6(40 \%)$ were females (male:female ratio of 3:2). The dichotomy reflected the nature of the heavy work of males in the agricultural society and higher susceptibility to trauma. Among the patients, 13 ( $86 \%$ of cases) had one lumbar disc operation 
before admission to hospital and 2 patients (14\%) had two operations. The primary procedures included discectomy with unilateral hemilaminectomy ( $\mathrm{n}=8,53 \%$ of cases) and discectomy with bilateral laminectomy (total laminectomy; $\mathrm{n}=7,47 \%$ ). The time from the primary surgery to recurrence averaged 5.7 years (range, 1.5-10 years). According to preoperative MRI (the investigative method of choice), the levels of recurrent disc herniation were L4-5 in 11 patients (8 cases ipsilateral and 3 cases contralateral), L5-S1 in 3 patients ( 2 cases ipsilateral and 1 contralateral), with one patient at L3-4 (ipsilateral) (Table 3). The most common postoperative complications (Table 4) were transient cerebrospinal fluid leakage in 2 patients (14\%), which were managed conservatively by absolute bed rest, tight bandage and frequent aspiration until complete resolution with no collection until the end of the followup period. Superficial wound infection developed in one patient $(6 \%)$ and was treated conservatively by bed rest, antibiotics and daily dressing until complete healing. By the end of the follow-up period, all patients had successful fusion in the form of lack of motion, lack of lucencies and formation of anterior bridging bone on flexion/extension X-rays with no failure rate or major complications related to either pedicle screws or cage. The mean JOA score showed significant improvement (Table 5), increasing from 9.5 on admission to 24.5 at the end of the study $(p<0.001)$. Outcome (Table 6) was excellent in 7 patients
(46\% of cases) with complete resolution of preoperative symptoms and return to daily activities at the end of the follow-up period, good in 6 patients (40\% of cases) with improvement of preoperative symptoms and sedentary life with restriction of heavy work. Excellent and good outcomes were considered a satisfactory outcome, and was reported in 13 patients ( $86 \%$ of cases). There were significant differences $(p<0.05)$ between patients presenting with recurrent disc at the ipsilateral side and those at the contralateral side. The latter group showed excellent outcome in all 4 patients (26\% of cases) compared with 3 patients (20\% of cases) in the ipsilateral group. This could

Table 3. Site of recurrent disc herniation

\begin{tabular}{lcc} 
Site or recurrence & Ipsilateral & Contalateral \\
\hline L4-5 & 8 & 3 \\
L5-S1 & 2 & 1 \\
L3-4 & 1 & 0 \\
\hline
\end{tabular}

Table 4. Postoperative complications in the studied group

\begin{tabular}{lc} 
Complications & No. $(\%)$ \\
Cerebrospinal fluid leakage & $2(14)$ \\
Wound infection & $1(6)$ \\
\hline Total & $3(20)$ \\
\hline
\end{tabular}

Table 5. Clinical outcome according to JOA score

\begin{tabular}{lccc} 
Mean JOA score & Preoperative & 24 months postoperative & $p$-value \\
Upper extremity function & 4 & 4 & $<0.001$ \\
Lower extremity function & 3 & 6 \\
Sensory function & 2 & 2 \\
Bladder function & 0.5 & 24 \\
\hline Total & 9.5 & \\
\hline
\end{tabular}

JOA, Japanese Orthopedic Association Scale.

Table 6. Surgical outcome according to the MacNab's classification

\begin{tabular}{lccc} 
Outcome & Ipsilateral group & Contalateral group & $p$-value \\
Excellent (>90\% improvement) & $3(3)$ & $4(26)$ & $<0.05$ \\
Good ( $75 \%-89 \%$ improvement) & $6(40)$ & 0 \\
\hline Fair ( $50 \%-74 \%$ improvement) & $2(14)$ & 0 \\
Poor (<50\% improvement) & 0 & $4(26)$ \\
\hline Total & $11(74)$ & 0 \\
\hline
\end{tabular}


be due to more adhesion around the previous discectomy site, which made dissection more difficult with increased risk of more complications.

\section{Discussion}

The progressive nature of degenerative diseases of the spine makes revision surgery at some point likely, even with optimal patient selection and surgical execution of patients who have previously undergone a decompressive lumbar laminectomy for herniated disc or lumbar stenosis [12]. The reported likelihood of reoperation after lumbar spine surgery may vary depending on the index procedure (discectomy or decompressive laminectomy), the size of the series, and the length of follow-up. Reoperation rates of $5 \%-11 \%$ are commonly reported. Studies with long-term follow-up (4-11 years) have reported that up to $28 \%$ of patients may undergo reoperation after a lumbar decompressive procedure for lumbar spinal stenosis [13].

Reoperations pose challenges in planning and execution that are distinct from those presented by primary surgery. Adhering to fundamental principles of patient evaluation and selection, surgical technique, and close follow-up evaluation will help to ensure optimal outcomes for patients undergoing revision laminectomy [14]. The optimal surgical approach for recurrent disc herniation remains debatable. Discectomy with fusion has several theoretical advantages. Specifically, interbody fusion reduces or eliminates segmental motion, immobilizes the spine, reduces mechanical stresses across the degenerated disc space and may reduce additional herniation at the affected disc space [15].

Revision spinal surgery is more challenging than primary surgery, owing to the indistinct anatomical planes and perineural scarring. It has been associated with high incidence of neurological injury, cerebrospinal fluid leakage and postoperative infection [16]. TLIF is an increasingly popular treatment for degenerative lumbar conditions. Its unilateral posterior approach enables anterior column stabilization and $360^{\circ}$ fusion with reduction of morbidity associated with PLIF and ALIF [17]. TLIF is similar to the more established PLIF in that it allows fusion without necessitating a separate anterior approach. Compared with PLIF, TLIF offers several advantages. It involves the unilateral removal of a facet joint, and this more lateral approach to the disc space requires minimal retraction of the dura mater, reducing the risk of neu-
Table 7. Preoperative clinical presentations in the study

\begin{tabular}{lc} 
Clinical presentations & No. $(\%)$ \\
\hline Low back pain and I sciatic pain & $15(100)$ \\
Sensory deficits & $6(40)$ \\
Motor deficits & $2(14)$ \\
\hline
\end{tabular}

rological injury. For the same reason, TLIF also can be performed at the upper lumbar levels where proximity to the conus medullaris makes the dural retraction necessary in PLIF unfeasible. In addition, TLIF can be performed contralateral to the site of a prior laminotomy, thus avoiding adherent scar tissue [18]. In our short study, we used TLIF as an optional surgical procedure in select cases of recurrent lumbar disc hernaition. This study included 15 patients with recurrent lumbar disc herniation fulfilling specific clinical and radiological criteria including presentation with severe back pain and unilateral sciatica after at least 6 months pain free-period postoperative, failure of conservative treatment for 3 months, progressive postoperative neurological deficit (Table 7), postoperative MRI showing recurrent posterolateral or lateral disc herniation at the same level. There were different primary surgical procedures included in this study as hemilaminectomy in 8 patients (54\% of cases) and total laminectomy in 7 patients ( $46 \%$ of cases). The average time from the primary surgery to recurrence was 6.5 years (range, 1-12 years). The most common level of recurrence reported was at $\mathrm{L}$ 4-5 level (74\%) and this correlates with prior observations [19] in a series of 36 patients; recurrence at the same level was observed in $75 \%$ of cases.

TLIF provides an approach through unilateral facetectomy to enter unscarred virgin tissue, which reduces the potential risk of dural tear and root injury. Limitations of this procedure include central disc herniations, disc calcification, redundant disc space, associated spondylodiscitis and osteoprotic patients. Only 2 patients (14\% of cases) developed postoperative transient cerebrospinal fluid leakage in our series and were treated conservatively by strict bed rest, tight bandage and antibiotics until complete resolution with no collection at the end of the follow-up period. Another study [20] reported a complication rate of $23 \%$ after repeated discectomy, with dural tear being the most common problem (18\% of patients). To overcome these complications, we suggest a more lateral entry point compared with PLIF, which can reduce dura and nerve root retraction and minimize the risk of neurological 
injury. We left central scar tissue untouched and a single cage packed with bone was impacted diagonally from the symptomatic side after proper dilatation of the disc space. This is similar to another study [21] that reported, as only unilateral facetectomy is required for the insertion of a single cage, the stiffness of the construction is significantly superior to the stand-alone two-cage analogue.

Bone grafting of the available surface area of the disc space is important for fusion success. Before cage insertion, the prepared laminectomy and facetectomy bone was grafted into the prepared disc space and in the cage. Because we used only one cage, there was more space for the bone graft than when two cages were inserted. The placement of additional bone grafts around the single cage may enhance the fusion rate; there were no pseudarthroses in our study. In our study, solid fusion was achieved in all cases at the final follow-up and this correlates with clinical results reported by others [22].

Regarding surgical outcome, 13 patients (86\% of cases) included in our study had satisfactory outcome as regarding improvement of preoperative manifestations with return to normal daily activities during the follow-up period with no major postoperative complications or neurological deficits. These results are comparable with the satisfactory clinical results previously reported [23].

\section{Conclusions}

TLIF with a single cage and excised local bone grafts in augmentation with bilateral pedicle screws seems to be an effective and affordable procedure with satisfactory clinical results for the treatment of selected cases of recurrent lumbar disc herniation. It can maintain the stability of the lumbar spine and has low complication rates, although further randomized, controlled, long-term evaluations and postoperative biomechanical analyses of the spine are necessary to confirm this.

\section{Conflict of Interest}

No potential conflict of interest relevant to this article was reported.

\section{References}

1. Fandino J, Botana C, Viladrich A, Gomez-Bueno J. Reoperation after lumbar disc surgery: results in 130 cases. Acta Neurochir (Wien) 1993;122:102-4.

2. Stewart G, Sachs BL. Patient outcomes after reoperation on the lumbar spine. J Bone Joint Surg Am 1996; 78:706-11.

3. Osterman H, Sund R, Seitsalo S, Keskimaki I. Risk of multiple reoperations after lumbar discectomy: a population-based study. Spine (Phila Pa 1976) 2003; 28:621-7.

4. Ray CD. Spinal interbody fusions: a review, featuring new generation techniques. Neurosurg Q 1997;7:13542.

5. Salehi SA, Tawk R, Ganju A, LaMarca F, Liu JC, Ondra SL. Transforaminal lumbar interbody fusion: surgical technique and results in 24 patients. Neurosurgery 2004;54:368-74.

6. Humphreys SC, Hodges SD, Patwardhan AG, Eck JC, Murphy RB, Covington LA. Comparison of posterior and transforaminal approaches to lumbar interbody fusion. Spine (Phila Pa 1976) 2001;26:567-71.

7. Benzel EC. Disc interspace distraction: is it really necessary? J Neurosurg 2003;99(2 Suppl):141-2.

8. Tribus CB. Circumferencial fusion techniques. In: Resnick DK, Haid RW; American Association of Neurological Surgeons, editors. Surgical management of low back pain. Rolling Meadows: American Association of Neurological Surgeons; 2001. p.117-21.

9. Potter BK, Freedman BA, Verwiebe EG, Hall JM, Polly DW Jr, Kuklo TR. Transforaminal lumbar interbody fusion: clinical and radiographic results and complications in 100 consecutive patients. J Spinal Disord Tech 2005;18:337-46.

10. Hirabayashi K, Miyakawa J, Satomi K, Maruyama T, Wakano K. Operative results and postoperative progression of ossification among patients with ossification of cervical posterior longitudinal ligament. Spine (Phila Pa 1976) 1981;6:354-64.

11. Papadopoulos EC, Girardi FP, Sandhu HS, et al. Outcome of revision discectomies following recurrent lumbar disc herniation. Spine (Phila Pa 1976) 2006;31:1473-6.

12. Javid MJ, Hadar EJ. Long-term follow-up review of patients who underwent laminectomy for lumbar stenosis: a prospective study. J Neurosurg 1998;89:17.

13. Martin BI, Mirza SK, Comstock BA, Gray DT, Kreuter W, Deyo RA. Reoperation rates following lumbar spine surgery and the influence of spinal fu- 
sion procedures. Spine (Phila Pa 1976) 2007;32:3827.

14. Bernard TN Jr. Repeat lumbar spine surgery: factors influencing outcome. Spine (Phila Pa 1976) 1993;18: 2196-200.

15. Vishteh AG, Dickman CA. Anterior lumbar microdiscectomy and interbody fusion for the treatment of recurrent disc herniation. Neurosurgery 2001;48:3347.

16. Reith C, Lausberg G. Risk factors of recurrent disc herniation. Neurosurg Rev 1989;12:147-50.

17. Lowe TG, Tahernia AD. Unilateral transforaminal posterior lumbar interbody fusion. Clin Orthop Relat Res 2002;(394):64-72.

18. Moskowitz A. Transforaminal lumbar interbody fusion. Orthop Clin North Am 2002;33:359-66.

19. Lehmann TR, LaRocca HS. Repeat lumbar surgery: a review of patients with failure from previous lumbar surgery treated by spinal canal exploration and lumbar spinal fusion. Spine (Phila Pa 1976) 1981;6:615-9.

20. Ebeling U, Kalbarcyk H, Reulen HJ. Microsurgical reoperation following lumbar disc surgery: timing, surgical findings, and outcome in 92 patients. J Neurosurg 1989;70:397-404.

21. Zhao J, Hai Y, Ordway NR, Park CK, Yuan HA. Posterior lumbar interbody fusion using posterolateral placement of a single cylindrical threaded cage. Spine (Phila Pa 1976) 2000;25:425-30.

22. Iida Y, Kataoka O, Sho T, et al. Postoperative lumbar spinal instability occurring or progressing secondary to laminectomy. Spine 1990;15:1186-9.

23. Cinotti G, Gumina S, Giannicola G, Postacchini F. Contralateral recurrent lumbar disc herniation: results of discectomy compared with those in primary herniation. Spine (Phila Pa 1976) 1999;24:800-6. 